Bundesgesundheitsbl 2020 • 63:361-367 https://doi.org/10.1007/s00103-019-03089-4 c) Springer-Verlag GmbH Deutschland, ein Teil von Springer Nature 2019
Stoffidentifikation, physikalische und chemische Eigenschaften

- IUPAC-Name: Benzen

- Synonyme: Benzol, Phen, Cyclohexatrien

- CLP-Index-Nr.: 601-020-00-8

- EG-Nr.: 200-753-7

- CAS-Nr.: 71-43-2

- Summenformel: $\mathrm{C}_{6} \mathrm{H}_{6}$

- Strukturformel:

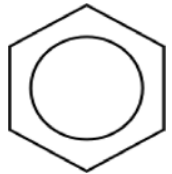

- Molekulargewicht: 78,11 g/Mol

- Schmelzpunkt: $5,5^{\circ} \mathrm{C}$

- Siedepunkt: $80,1^{\circ} \mathrm{C}$

- Dampfdruck: $10 \mathrm{kPa}$ (bei $20^{\circ} \mathrm{C}$ )

- Wasserlöslichkeit: $1,8 \mathrm{~g} / \mathrm{l}$ (bei $25^{\circ} \mathrm{C}$ )

- Log $\mathrm{P}_{\text {Octanol/Wasser }}: 2,13$

- Umrechnung $\left(20^{\circ} \mathrm{C}, 1013 \mathrm{hPa}\right): 1 \mathrm{ml} /$ $\mathrm{m}^{3}=3,25 \mathrm{mg} / \mathrm{m}^{3} ; 1 \mathrm{mg} / \mathrm{m}^{3}=0,31 \mathrm{ml} /$ $\mathrm{m}^{3}$; (Daten aus $\left.[9,66]\right)$

\section{Exposition}

Benzol ist ein natürlicher Bestandteil des Erdöls und wird zur Herstellung einer Vielzahl chemischer Verbindungen (z. B. Styrol, Cumol, Cyclohexan, Nitrobenzol) und daraus abgeleiteter Folgeprodukte eingesetzt. Weltweit beträgt die Produktion 2012 ca. 43 Mio. t, in Westeuropa wird 2014 von 6,7-7,5 Mio. t ausgegangen [22].

Wesentliche Quellen in der Umwelt sind unvollständige Verbrennungsvorgänge (z. B. beim Betrieb von Verbren-

Bekanntmachung des Umweltbundesamtes

\title{
Vorläufiger Leitwert für Benzol in der Innenraumluft
}

\section{Mitteilung des Ausschusses für Innenraumrichtwerte}

nungsmotoren, Waldbrände und Vulkanausbrüche). In Innenräumen tragen Tabakrauchen, Räucherstäbchen, Heimwerkerartikel, der Betrieb von Kaminen und Öfen, die Außenluft (insbesondere in verkehrsreichen Bereichen) und ggf. unmittelbar angrenzende Garagen zur Benzol-Exposition bei [15, 65, 67]. Andere Quellen wie Lebensmittel oder Trinkwasser spielen eine untergeordnete Rolle [54]. So schätzte 1993 die WHO die Zufuhr von Benzol über Nahrungsmittel (einschl. Trinkwasser) in den USA und Kanada mit höchsten 1,4 $\mu \mathrm{g} / \mathrm{Tag}$ ein
[24]. In einer aktuelleren belgischen Untersuchung von 455 Lebensmittelproben konnte Benzol in 58 \% der Proben, insbesondere in geräuchertem Fisch in Dosen, oberhalb der Bestimmungsgrenze quantifiziert werden [35]. Die mittlere tägliche Aufnahme über alle Nahrungsmittelgruppen errechnete sich unter Verwendung des „upper bound“ in dieser Studie zu $0,020 \mu \mathrm{g} / \mathrm{kg}$ Körpergewicht $(97,5$. Perzentil: $0,078 \mu \mathrm{g} / \mathrm{kg} \mathrm{KG).}$

\section{Tab. 1 Vorkommen von Benzol in der Luft von Gebäuden in Deutschland}

\begin{tabular}{|c|c|c|c|c|c|}
\hline Innenraum/Zeitraum & $N$ & $\begin{array}{l}\text { Median } \\
{\left[\mu \mathrm{g} / \mathrm{m}^{3}\right]}\end{array}$ & $\begin{array}{l}\text { 95. Perz. } \\
{\left[\mu \mathrm{g} / \mathrm{m}^{3}\right]}\end{array}$ & $\begin{array}{l}\text { Maximum } \\
{\left[\mu \mathrm{g} / \mathrm{m}^{3}\right]}\end{array}$ & Referenz \\
\hline \multicolumn{6}{|l|}{ Wohnungen } \\
\hline 1985-1986 & 479 & 7,2 & 22 & 90 & UBA 1991 [57] \\
\hline 2003-2006 & 555 & 1,8 & 7,7 & 61 & UBA 2008 [58] \\
\hline 2006-2012 & 717 & 1,0 & 4,0 & - & $\begin{array}{l}\text { Hofmann et al. } \\
2014 \text { [20] }\end{array}$ \\
\hline 2015-2017 & 620 & 1,1 & 4,5 & 29 & UBA 2019 [60] \\
\hline \multicolumn{6}{|l|}{ Gemeinschaftsräume } \\
\hline $\begin{array}{l}\text { Schulen + Kitas, } \\
\text { 1990-1993 }\end{array}$ & 395 & 1,5 & 5,0 & - & UfU 1994 [61] \\
\hline Schulen, 2004-2005 & 165 & 0,3 & 4,0 & 8,0 & $\begin{array}{l}\text { Fromme et al. } \\
2008 \text { [14] }\end{array}$ \\
\hline $\begin{array}{l}\text { Schulen + Kitas, } \\
\text { 2005-2007 }\end{array}$ & 285 & $<1,0$ & 2,0 & 5,0 & LasD 2009 [31] \\
\hline Schulen, 2006-2012 & 499 & 1,0 & 3,1 & - & $\begin{array}{l}\text { Hofmann et al. } \\
2014 \text { [20] }\end{array}$ \\
\hline Kitas, 2011-2012 & 63 & 2,0 & 4,3 & 5,9 & $\begin{array}{l}\text { Fromme et al. } \\
2008 \text { [14] }\end{array}$ \\
\hline \multicolumn{6}{|l|}{ Büro } \\
\hline 2006-2012 & 1769 & 1,0 & 4,0 & - & $\begin{array}{l}\text { Hofmann et al. } \\
2014 \text { [20] }\end{array}$ \\
\hline
\end{tabular}




\section{Innenraumluft}

Die Belastung der Luft von Innenräumen in Deutschland mit Benzol ist in der $\bullet$ Tab. 1 zusammengestellt. Eine wesentliche Einflussgröße im Hinblick auf die Benzol-Konzentrationen in der Innenraumluft stellen die abnehmenden Benzol-Konzentrationen in der Außenluft (s. Abschn. 2.2) dar. Während im Innenraum in den achtziger und neunziger Jahren ein deutlicher Rückgang der Belastung stattgefunden hat, fiel er in den letzten Jahren nur noch gering aus. So beobachteten Hoffmann et al. [20] in Innenräumen einen Rückgang der Mediane von $2,0 \mu \mathrm{g} / \mathrm{m}^{3}$ in 2002 auf $0,5 \mu \mathrm{g} / \mathrm{m}^{3}$ in 2012 und des 95 . Perzentils von 7,0 auf $3,0 \mu \mathrm{g} / \mathrm{m}^{3}$.

\section{Außenluft}

Die Benzol-Konzentrationen in der AuBenluft sind in den letzten Jahrzehnten um etwa eine Größenordnung gesunken, vor allem durch die Begrenzung des Benzol-Gehaltes in Kraftstoffen Ende der 1990er Jahre [9]. Seit 2010 gilt für Benzol ein Jahresmittelgrenzwert in der Außenluft in Höhe von $5 \mu \mathrm{g} / \mathrm{m}^{3}$ [1]. Dieser wird in Deutschland seit 2014 an allen Messstationen der Länder und des Bundes unterschritten [59]. In der Außenluft zeigen sich deutliche Jahresschwankungen mit höheren Benzol-Konzentrationen im Winter als im Sommer $[57,66]$.

\section{Innere Exposition}

Die am besten geeigneten Biomarker einer nicht-gewerblichen internen Exposition der Bevölkerung stellen Benzol, S-Phenylmercaptursäure (SPMA) und t,tMuconsäure im Harn (siehe auch Abschn. 3) dar. Auf der Basis von drei deutschen Studien ergibt sich für SPMA im Urin der nichtrauchenden Allgemeinbevölkerung ein 95 . Perzentil zwischen 0,3 und $0,5 \mu \mathrm{g}$ SPMA/g Kreatinin [29]. Die DFGArbeitsgruppe legte 2016 auf der Basis einer italienischen Studie von Campagna et al. [5] an 86 Nichtrauchern für Benzol im Harn 0,3 $\mu \mathrm{g}$ SPMA/g Kreatinin als Biologischen Arbeitsplatzreferenzwert (BAR) fest [29]. Für Benzol im Harn wurde ein BAR-Wert von $0,3 \mu \mathrm{g} / \mathrm{l}$ Harn und für die
t,t-Muconsäure ein BAR von $150 \mu \mathrm{g} / \mathrm{g}$ Kreatinin abgeleitet.

Aktuell wurden in der Deutschen Umweltstudie zur Gesundheit von Kindern und Jugendlichen (GerES V) repräsentative Daten für die in Deutschland lebenden Kinder und Jugendliche im Alter von 3-17 Jahren erhoben. Das 95. Perzentil für SPMA beträgt $0,33 \mu \mathrm{g}$ SPMA/g Kreatinin und der Median 0,08 $\mu \mathrm{g}$ SPMA/ g Kreatinin für nichtrauchende Kinder und Jugendliche [59]. Nur $2 \%$ der Teilnehmenden lagen unterhalb der Bestimmungsgrenze von $0,02 \mu \mathrm{g} / \mathrm{l}$.

\section{Toxikokinetik}

In einer Studie, in der 3 weibliche und 3 männliche Probanden über $4 \mathrm{~h}$ gegenüber $169-202 \mathrm{mg} / \mathrm{m}^{3}$ exponiert waren, wurde stündlich der aufgenommene, ausgeatmete und im Körper verbliebene Benzol-Anteil bestimmt [38]. Der im Körper verbleibende Anteil nahm für beide Geschlechter im Laufe der vier Stunden ab, wobei der verbleibende Anteil bei Frauen in den ersten zwei Stunden signifikant höher war. Nach vier Stunden wurde zwischen den Geschlechtern kein Unterschied mehr festgestellt. Die finalen mittleren Werte für Aufnahme, Ausscheidung und Verbleib betrugen 47,17 und $30 \%$. In einer vorherigen Studie, in der die Aufnahme bei Konzentrationen zwischen 195 und $260 \mathrm{mg} / \mathrm{m}^{3}$ untersucht wurde, bestimmten die Autoren auch nach vier Stunden eine signifikant unterschiedlich Aufnahme: Männer $(n=6) 20 \%$ und Frauen $(n=6)$ $42 \%$. In einer weiteren Studie, in der 3 männliche Probanden über $4 \mathrm{~h} 30 \mathrm{mg} / \mathrm{m}^{3}$ oder $5,2 \mathrm{mg} / \mathrm{m}^{3}$ einatmeten, ergab sich eine Aufnahme von $48 \%$ bei der hohen Konzentration und $52 \%$ bei der niedrigeren [39]. Bei der Exposition von 7 Probanden gegenüber BTX-Aromaten mit online gemessenen Benzol-Konzentrationen von ca. $0,013 \mathrm{mg} / \mathrm{m}^{3}$ wurde im Mittel ein respiratorischer Absorptionsfaktor von $55 \%$ beobachtet (3 Frauen: 66,5\% und 4 Männer 46,4\%) [21]. Auch eine dermale Aufnahme von Benzol bei Exposition gegenüber kontaminierter Luft ist grundsätzlich möglich, wird aber als vernachlässigbar eingeschätzt. So kommen Rauma et al. [46] auf der Basis von drei tierexperimentellen Studien an Schweinen, Ratten und
Mäusen, zu dem Schluss, dass der mittlere dermale Anteil an der Gesamtaufnahme bei ca. 2,9\% liegt.

Der größte Teil des inhalierten Benzols wird auch wieder als Benzol abgeatmet. Absorbiertes Benzol wird metabolisiert und im Wesentlichen über den Urin ausgeschieden [7].

Die Verstoffwechselung von Benzol ist sehr komplex und beginnt mit der Oxidation mittels Cytochrom P450, hauptsächlich CYP2E1 zu Benzoloxid (Epoxid). CYP2E1wird vor allem in der Leber aber auch in anderen Organen exprimiert [2]. Da CYP2E1auch im Knochenmark vorkommt, kann Benzol dort auch direkt metabolisiert werden [9]. Benzoloxid liegt im Gleichgewicht mit Oxacycloheptatrien („Oxepin“) vor, das sich überwiegend spontan in Phenol umlagert. Unter weiterer Oxidation können 1,2-Phendiol ("Catechol") und 1,4-Phendiol („Hydrochinon“) sowie 1,2,4-Phentriol („Benzoltriol $\left.{ }^{\prime}\right)$ entstehen. Benzoloxid bzw. Oxacycloheptatrien kann mit Glutathion die harngängige S-Phenylmercaptursäure (SPMA) bilden oder unter Ringöffnung in trans-trans-Hexadiendial („Muconaldehyd") zerfallen, das zur trans-trans-Hexadiendisäure („t-t-Muconsäure") oxidiert wird. In einem Seitenweg kann 1,2-Phendiol auch aus Benzoloxid über 1,2-Phendihydrodiol gebildet werden. Mithilfe von Myeloperoxidasen, die vor allem in neutrofilen Granulozyten vorkommen, können aus den beiden Phendiolen die entsprechenden Phendione („Benzochinone") gebildet werden. Enzympolymorphismen sind für die Glutathion-STransferase (GST), CYP2E1 und für die Quinonoxidoreduktase NQO1 bekannt.

Bei Ratten und Mäusen wird Benzol überwiegend ( $57 \%$ bzw. $37 \%$ ) als sulfatiertes bzw. glucuronidiertes Phenol ausgeschieden, während t-t-Muconsäure und SPMA ca. $20 \%$ bzw. 1-1,5\% ausmachen $[52,53]$. Bei 25 Arbeitern erfolgte 70-88 \% der Ausscheidung im Harn als Phenol bzw. seinen Konjugaten, gefolgt von t-t-Muconsäure, während nur 0,05$0,3 \%$ des aufgenommenen Benzols als SPMA ausgeschieden wurden [40]. Die Eliminationshalbwertzeit von SPMA beträgt 9-13 h [7]. In einer Untersuchung an 11 exponierten Arbeitern (Median in der Luft ca. 26 ppm) wurden Halbwertszeiten 
von $13 \mathrm{~h}$ für SPMA und $14 \mathrm{~h}$ für die t-tMuconsäure gefunden [40]. Zwischen Individuen wurden deutliche Unterschiede in der Elimination der Metaboliten gefunden, was auf interindividuelle Unterschiede in der Effizienz Benzol zu metabolisieren hinweist [41]. In weiteren Analysen wurde der Einfluss von Polymorphismen in metabolisch relevanten Genen untersucht: CYP2E1, Quinonoxidoreduktase (NQO1), GSTT1 und Myeloperoxidase (MPO). Nur beim GSTT1-Gen wurde der Effekt festgestellt, dass ein Null-Allel die Bildung von SPMA halbieren kann [42]. Insbesondre die reaktiven Metaboliten Catechol, Chinone und Hydrochinone werden im Tierexperiment nur langsam aus dem Knochenmark ausgeschieden [16]. Für die Hauptmetabolite wurde im Konzentrationsbereich zwischen $0,088 \mathrm{mg} /$ $\mathrm{m}^{3}$ und ca. $100 \mathrm{mg} / \mathrm{m}^{3}$ eine dosisabhängige Abnahme der Ausscheidung beobachtet [26, 41, 45]. In weiteren Studien (263 Frauen) untersuchte Rappaport et al. [44] die Ausscheidungskinetik von Benzolmetaboliten nach inhalativer Exposition. Die Ergebnisse weisen auf zwei parallele Stoffwechselpfade mit unterschiedlicher Affinität hin, mit CYP2E1 im niedrig-affinen Pfad, der vor allem bei höheren Konzentrationen relevant ist. Der hoch-affine Pfad mit sehr beschränkter Kapazität, der insbesondere bei niedrigen Konzentrationen $\left(<1 \mathrm{ml} / \mathrm{m}^{3}\right)$ relevant zu sein scheint wurde bislang nicht identifiziert. Dieses 2-Wege-Modell weist im Expositionsbereich unter $1 \mathrm{ppm}$ eine ca. 3-fach höhere Metabolisierungsrate als das Modell mit nur einem Stoffwechselpfad [2].

\section{Wirkungen}

Benzol gehört zu den toxikologisch sehr gut untersuchten Stoffen. Angesichts des komplexen Wirkungsmechanismus von Benzol sind dennoch Wissenslücken vorhanden. Verschiedene wissenschaftliche Organisationen haben eine Zusammenschau des bisherigen Kenntnisstandes veröffentlicht $[2,7,9,22,62,66]$. Bei wiederholter Exposition besteht eine klare wissenschaftlich Evidenz aus Tierversuchen und epidemiologischen Studien, dass Benzol das Knochenmark und hier das blutbildende System und auch das Immunsystem beeinträchtigt. In diesem
Zusammenhang treten insbesondere Anämien, Leukozytopenien und Thrombozytopenien auf. In umfangreichen bevölkerungsbezogenen Studien wurde eine Assoziation zwischen Benzol und Leukämien, insbesondere akut myeloische Leukämie, gesehen. Hinsichtlich des Wirkungsmechanismus der Krebsentstehung, der wahrscheinlich sehr komplex ist und sowohl primäre als auch sekundär gentoxische Effekte umfasst, bestehen Unsicherheiten. Neben der Adduktbildung durch reaktive Metabolite müssen insbesondere epigenetische Wirkungsmechanismen wie oxidativer Stress durch Bildung von Phendiol bzw. Phendion und eine Hemmung der Topoisomerase II, für die eine Schwelle angenommen werden kann, berücksichtigt werden. Akute Effekte auf das Nervensystem, die Leber oder Niere traten in der Vergangenheit nur bei sehr hohen BenzolKonzentrationen von oberhalb etwa von $100 \mathrm{mg} / \mathrm{m}^{3}$ auf [9].

\section{Hämatotoxizität}

Als Mechanismus der hämatotoxischen Effekte wird die Bildung von freien Radikalen durch verschiedene Metabolite (z. B. das 1,2-Benzochinon) angesehen, In zahlreichen Studien an Beschäftigten mit Expositionen oberhalb von $33 \mathrm{mg}$ Ben$\mathrm{zol} / \mathrm{m}^{3}$ nahm die Anzahl der roten Blutkörperchen (Anämie), der Leukozyten (Leukozytopenie) und der Blutplättchen (Thrombozytopenie) ab [9]. Ursächlich für diese Panzytopenie ist eine aplastische Anämie, aus der sich eine Leukämie entwickeln kann.

\section{Gentoxizität}

Die in vitro Mutagenitätstests an Bakterien und Hefen ergaben mit und ohne metabolische Aktivierung überwiegend negative Ergebnisse. Die in vitro Untersuchungen an Human- und Säugerzellen zeigten dahingehend ein durchwachsenes Bild von positiven und negativen Befunden. Die in vivo Studien an Nagetieren waren mit wenigen Ausnahmen durchgehend positiv in Hinsicht auf alle relevanten genotoxischen Endpunkte wie Mikrokernbildung, Chromosomenaberrationen, Aneuploidie, Schwesterchromatidaustausch, DNAStrangbrüche und Genmutationen.
Eine wichtige Grundlage für die Beurteilung des genotoxischen Potentials von Benzol stellen zahlreiche Studien an Beschäftigten von Tankstellen, Raffinerien, Schuh- und Klebstofffabriken und anderen Industriebereichen dar. Im Comet Assay wurde die DNA Schädigung in Blutzellen wie Blutlymphozyten bereits bei der Exposition im Bereich von 0,035-3,5 mg/ $\mathrm{m}^{3}$ nachgewiesen [9]. Die ECHA-RAC bewertet jedoch die DNA Schädigung lediglich als Indikator für die Genotoxizität, da sie den Mutationen nicht gleichgestellt werden können.

Maßgeblich für die Toxizität von Benzol werden klastogene und aneugene Wirkungen angesehen. Bezüglich Klastogenität und Aneugenität bewertet ECHA die humanen Befunde in Abhängigkeit von der Konzentration. Die Wirkungen waren eindeutig positiv in Studien mit Konzentrationen ab $3 \mathrm{mg} / \mathrm{m}^{3}$. Der ECHA nach waren die Ergebnisse im Bereich von 0,1-1 ppm uneinheitlich und weniger belastbar. In dem niedrigeren Dosisbereich (unter 0,1 ppm) hält ECHA die positiven Studien als nicht belastbar auf Grund von Mängeln bei der Expositionsabschätzung, Koexposition mit polyzyklischen aromatischen Kohlenwasserstoffen oder möglichen dermalen Expositionen [9]. Die Ergebnisse der belastbaren Studien waren negativ.

Zusätzlich zu genotoxischen Wirkungen wurden für Benzol auch epigenetische Veränderungen in Form von der DNA Methylierung, Histonmodifikationen und nicht-kodierenden RNAs beschrieben. Die Bedeutung dieser Befunde für die Benzol Toxizität ist derzeit für deren gesundheitliche Bewertung jedoch nicht ausreichend untersucht.

\section{Kanzerogenität}

Aus epidemiologischen Studien an Beschäftigten ergaben sich eindeutige Hinweise auf einen kausalen Zusammenhang zwischen einer beruflichen Benzol-Exposition und einem erhöhten Risiko einer akuten myeloischen Leukämie (AML). Unter dem Begriff AML wird eine Gruppe biologisch heterogener Erkrankungen mit einer Vielzahl zytogenetischer Aberrationen zusammengefasst. Bei fast allen Patienten (>99\%) ließ sich molekulargenetisch mindestens eine von 27 Treiber- 
genmutationen identifizieren, im Mittel lagen vier Mutationen vor [36]. AML ist vor allem eine Erkrankung älterer Menschen: der Altersmedian der Erkrankung liegt bei über 70 Jahren [12].

Darüber hinaus ergaben sich Hinweise auf einen Zusammenhang mit dem myelodysplastischen Syndrom (MDS) sowie weiteren Leukämieunterarten (ALL, CML, CLL), Non-Hodgkin-Lymphomen (NHL) und multiplem Myelom [32, 33]. Während der Zusammenhang zwischen Benzolexposition und AML-Entstehung als gesichert gilt, wird er für die anderen Tumorformen von der IARC als noch begrenzt angesehen [9].

Etliche epidemiologische Studien gingen der Frage nach, ob eine Exposition von Schwangeren gegenüber Benzol in ihrer Umwelt mit einem erhöhten LeukämieRisiko ihrer Kinder verknüpft sein könnte (z. B. $[6,23,25,27,34,43,55])$. Bei einigen Studien zeigte sich ein signifikanter $\mathrm{Zu}$ sammenhang, insgesamt sind die Ergebnisse jedoch widersprüchlich. Insbesondere wurde nicht immer zwischen AML und akuter lymphatischer Leukämie (ALL) unterschieden; letztere stellt etwa $80 \%$ der Leukämien im Kindesalter, vor allem im 1.-5. Lebensjahr. Lediglich in drei FallKontrollstudien aus Italien, Kalifornien und Dänemark wurde bisher eine Assoziation zwischen kindlicher AML und Benzolexposition untersucht $[18,19,64]$, der auch in einer Metaanalyse als statistisch bedeutsam, beim Fehlen einer Schwelle, angesehen wurde [13]. Auch für 1989 dänische Kinder mit Leukämien ließ sich eine Tendenz für einen Zusammenhang zwischen Benzolexposition im Kindesalter und AML, nicht aber ALL, und der modellierten Außenluftbelastung finden [43]. Belastbare Erhebungen zur Exposition gegenüber Benzol fehlen zumeist: die Exposition wurde teilweise telefonisch erfragt oder aus stationären Außenluftdaten abgeschätzt, selten wurden personenbezogene Messungen der Exposition durchgeführt. Die bisherigen Ergebnisse lassen deshalb keine abschließende Bewertung zu.

\section{Reproduktionstoxizität}

Belastbare Humanstudien zur Reproduktions- und Entwicklungstoxizität von Benzol liegen nicht vor. Lediglich eini- ge Studien wurden bisher publiziert, deren Aussagekraft durch eine geringe Probandenanzahl, keine Benzolmessungen oder fehlende Hinweise zu Koexposition mit anderen Schadstoffen begrenzt ist [9]. Tierexperimentell wurde bei verschiedenen Untersuchungen an Mäusen und Ratten keine reproduktionstoxischen Effekte bei Benzol-Konzentrationen unter 130$160 \mathrm{mg} / \mathrm{m}^{3}$ beobachtet.

\section{Geruchswahrnehmung}

Nagata [37] nennt für Benzol eine Geruchswahrnehmungskonzentration ODT $_{50}$ von $9 \mathrm{mg} / \mathrm{m}^{3}$. Weitere Daten sind in der wissenschaftlichen Literatur bisher nicht veröffentlicht.

\section{Bewertung}

\section{Einstufungen/Bestehende Regelungen}

Benzol ist auf der Basis der Verordnung (EG) Nr. 1272/2008 in der EU als bekanntermaßen krebserzeugend für den Menschen (Carc. 1A) und als erbgutverändernd (Muta. 1B) eingestuft [8].

Der verbindliche EU-Arbeitsplatzgrenzwert für Benzol beträgt 3,25 mg/m 3 [10]. Unabhängig davon haben zwischenzeitlich einzelne Mitgliedsstaaten niedrigere Arbeitsplatzgrenzwerte zwischen 0,7 und $1,5 \mathrm{mg} / \mathrm{m}^{3}$ eingeführt [9].

Nach Ansicht des deutschen Ausschusses für Gefahrstoffe (AGS) sind mehrere Mechanismen bei der benzolverursachten Leukämieentstehung relevant. Dies weist laut AGS auf eine mögliche Nichtlinearität bei Extrapolation in den Niedrigrisikobereich hin [2]. Der AGS zog für seine Risikoabschätzung deshalb sowohl ein lineares als auch ein sublineares Modell heran. Angesichts des nicht völlig geklärten Wirkungsmechanismus von Benzol [17] wählte der AGS vorsorglich ein lineares Modell. In die Risikobetrachtung gingen 13 Arbeitsplatzstudien ein, aus denen sich eine mittlere Effektkonzentration von 582 ppm-Jahre für ein zusätzliches Leukämierisiko von $10 \%$ $\left(\mathrm{ED}_{10}\right)$ ergab. Dies entspricht bei 40 Arbeitsjahren einer mittleren Konzentration von $47 \mathrm{mg} / \mathrm{m}^{3}$. Für das Toleranzrisiko $\left(4 \times 10^{-3}\right)$ und Akzeptanzrisiko $\left(4 \times 10^{-4}\right)$ ergaben sich damit Konzentrationen von 1,9 bzw. $0,2 \mathrm{mg} / \mathrm{m}^{3}$.

Da EU-SCOEL bislang keinen Arbeitsplatzgrenzwert für Benzol vorgelegt hatte [11], hatte die EU-Kommission im März 2017 den Risikobewertungsausschuss der Europäischen Chemikalienagentur (ECHA-RAC) gebeten, einen Arbeitsplatzgrenzwert für Benzol abzuleiten [9]. Er war bei seiner Ableitung Benzol davon aus, dass überwiegend die gebildeten Metabolite im Knochenmark und im Blut für die chromosomale Veränderungen verantwortlich gemacht werden können, aus denen sich letztlich Leukämien entwickeln. Zur Ableitung eines Grenzwertes zog ECHA-RAC die Endpunkte Hämatotoxizität sowie die klastogene und aneugene Wirkung von Benzol heran. Für die Hämatotoxizität bei Beschäftigten ging ECHA-RAC von einer LOAEC von 6,5 $\mathrm{mg}$ Benzol $/ \mathrm{m}^{3}$ aus. Angesichts einer hohen Anzahl untersuchter Beschäftigter (z. B. Tsai et al. [56]: $n=1200$, Koh et al. [28]: $n=10.700$ ), die auch Personen mit Polymorphismus einschloss, hielt ECHARAC einen Intraspeziesfaktor von 1 für ausreichend. Da es sich um Langzeitstudien handelte, wurde ein Faktor von 1 für die Expositionsdauer verwendet. Für die Extrapolation von der LOAEC zur NAEC hielt ECHA-RAC einen Faktor von 3 für ausreichend, da die Effekte an der LOAEC gering waren und innerhalb des Normbereiches lagen. Damit ergab sich für den Endpunkt Hämatotoxizität eine NAEC von 6,5: [ $1 \times 1$ $\times 3]=2,2 \mathrm{mg} / \mathrm{m}^{3}$. Klastogene und aneugene Effekte traten nach Ansicht des ECHARAC in den meisten Langzeitstudien erst bei oder oberhalb von $3,25 \mathrm{mg} / \mathrm{m}^{3}$ auf (LOAEC). Bei diesem Kenntnisstand hielt ECHA-RAC angesichts der hohen Fallzahlen (s. oben) einen Intraspeziesfaktor von 2 für ausreichend. Für die Extrapolation von der LOAEC zur NAEC hielt ECHA-RAC wegen der Schwere des Effekts einen Faktor von 10 für angemessen. Daraus folgte eine NAEC von $3,25 \mathrm{mg} / \mathrm{m}^{3}$ : $[2 \times 10]=$ (gerundet) $0,16 \mathrm{mg} / \mathrm{m}^{3}$. Im Vergleich der beiden Endpunkte schlug ECHA-RAC der EUKommission diese Konzentration als Arbeitsplatzgrenzwert vor [9].

Zum Schutz der Allgemeinbevölkerung hatte die US-Umweltschutzbehörde (USEPA) auf der Basis einer kleineren $(n=44)$ chronischen Arbeitsplatzstudie [50] für den Endpunkt Abnahme der Lymphozy- 
tenzahl im Blut und Anpassung an eine kontinuierliche Exposition eine $\mathrm{BMCL}_{1 \mathrm{SD}}$ von $8,2 \mathrm{mg} / \mathrm{m}^{3}$ abgeschätzt und mit einem Gesamtextrapolationsfaktor von 300 (Faktor 10 für BMCL zu NOAEC, 10 für Intraspezies-Unterschiede, Faktor 3 für Datenmängel) eine Referenzkonzentration für Benzol von 0,03 mg/m $\mathrm{m}^{3}$ abgeleitet [63]. Unter Berücksichtigung des Unit Risks, für das eine Spannweite von $2,2 \times 10^{-6}$ bis $7,8 \times 10^{-6}$ pro $\mu \mathrm{g} / \mathrm{m}^{3}$ angegeben wurde, würde die Referenzkonzentration einem Leukämierisiko von $6,6 \times 10^{-5}$ bis $2,3 \times 10^{-4}$ entsprechen.

Die US-Behörde für Giftstoffe und Krankheitsregistrierung (ATSDR) hat drei Richtwerte (sogenannte minimal risk level) für adverse nicht kanzerogener Effekte abgeleitet. Für den Kurzzeitrichtwert wurde eine Inhalationsstudie an Mäusen (6 Tage mit je $6 \mathrm{~h} / \mathrm{Tag}$ ) herangezogen, bei der auch bei der niedrigsten Konzentration von $33 \mathrm{mg} / \mathrm{m}^{3}$ die Lymphozyten-Proliferation nach Mitogen-Stimulation reduziert war [51]. Nach Anpassung an eine kontinuierliche Exposition und Verwendung eines Unsicherheitsfaktors von 10 für die Verwendung eines LOAECs statt NOAECs, eines Interspeziesfaktors von 3 und eines Intraspeziesfaktors von 10 ergab sich ein Kurzzeitrichtwert (bis 2 Wochen) von $0,03 \mathrm{mg} /$ $\mathrm{m}^{3}$. Basis für den intermediären Richtwert war eine subakute Inhalationsstudie an Mäusen (28 Tage, 5 Tage/Woche, 6 h/Tag) [49], bei der signifikant verzögerte Reaktionen der Milzlymphozyten auf fremde Antigene ab der niedrigsten Konzentration von $31 \mathrm{mg} / \mathrm{m}^{3}$ bestimmt wurden. Nach Anpassung an eine kontinuierliche Exposition und Verwendung eines Unsicherheitsfaktors von 10 für die Verwendung eines LOAECs statt NOAECs, eines Interspeziesfaktors von 3 und eines Intraspeziesfaktors von 10 ergab sich ein intermediärer Richtwert (15-364 Tage) von 0,02 $\mathrm{mg} / \mathrm{m}^{3}$ abgeleitet. Der Langzeitwert ( $a b$ ein Jahr) von $0,01 \mathrm{mg} / \mathrm{m}^{3}$ basiert auf der Arbeitsplatzstudie von Lan et al. [30]. Für die signifikante Abnahme der B-Lymphozyten bei den exponierten Arbeitern wurde eine $\mathrm{BMCL}_{0,25 \mathrm{sd}}$ modelliert und anschließend auf eine kontinuierliche Exposition extrapoliert (8/24 h und 6/7 Tagen) [62].

Die französische Behörde für Lebensmittelsicherheit, Umwelt und Arbeit hat die drei Richtwerte für Benzol der USATSDR [62] für die Bewertung der In- nenraumluft übernommen. Sie weist ergänzend darauf hin, dass eine mit einem Risiko von $10^{-6}$ verbundene lebenslange Exposition einer Konzentration von $0,0002 \mathrm{mg} / \mathrm{m}^{3}$ und eine mit einem Risiko von $10^{-5}$ verbundene einer Konzentration von $0,002 \mathrm{mg} / \mathrm{m}^{3}$ entsprechen würde [4]. Damit entspräche der französische Langzeitrichtwert von $0,01 \mathrm{mg} \mathrm{Benzol} / \mathrm{m}^{3}$ einem Leukämierisiko von $5 \times 10^{-5}$.

Das niederländische Reichsinstitut für Volksgesundheit und Milieuhygiene (RIVM) hat für Benzol als gentoxisches Kanzerogen das maximal zulässige Risiko (Maximum Permissible Risk - MPR) von $10^{-4}$ festgelegt, was bei lebenslanger Exposition einer Innenraumluftkonzentration von $0,02 \mathrm{mg} / \mathrm{m}^{3}$ entspricht [47]. Dieser Wert basiert auf der Bewertung der EU-Arbeitsgruppe für Benzol in der $\mathrm{Au}$ ßenluft, wonach der geometrische Mittelwert der Risikoschätzer für Leukämie bei $6 \times 10^{-6}$ für $1 \mu \mathrm{g} / \mathrm{m}^{3}$ Benzol liegt [48].

\section{Vorläufiger Leitwert für Benzol in der Innenraumluft}

Nach Auffassung des Ausschusses für Innenraumrichtwerte müssen zur gesundheitlichen Bewertung eines krebserzeugenden Stoffes in der Innenraumluft belastbare Angaben zu seinem üblichen Vorkommen in der Innenraumluft in Form des 95. Perzentils (Referenzwert) sowie zur Expositions-Risikobeziehung vorliegen [3]. Folgende Bewertung soll dann erfolgen:

Es wird diejenige Konzentration des krebserzeugenden Stoffes in der Innenraumluft ermittelt, die nach lebenslanger Exposition mit einem theoretischen Krebsrisiko von $10^{-6}$ verbunden ist. Diese Konzentration soll mit dem Referenzwert der Substanz verglichen werden. Liegt die aus der Expositions-Risikobeziehung abgeleitete Konzentration oberhalb des möglichst aktuellen Referenzwertes, wird sie als risikobezogener Leitwert für die Bewertung verwendet. Ist die Konzentration des Referenzwertes mit einem höheren theoretischen Risiko als $10^{-6}$ verknüpft, wird ein vorläufiger Leitwert anhand des Referenzwertes in der Innenraumluft festgelegt.

Der Ausschuss für Gefahrstoffe kommt im Rahmen seiner Festlegung einer Expositions-Risikobeziehung zu einem Risiko- wert von $4 \times 10^{-5}$ bei $0,02 \mathrm{mg} / \mathrm{m}^{3}$ [2]. Wenn diese Relation auf die Bedingungen einer lebenslangen Exposition der allgemeinen Bevölkerung umgerechnet wird 5,7 $\left(20 \mathrm{~m}^{3} \mathrm{~d}^{-1} /\right.$ $\left.10 \mathrm{~m}^{3} \mathrm{~d}^{-1} \times 7 \mathrm{~d} / 5 \mathrm{~d} \times 52 \mathrm{w} / 48 \mathrm{w} \times 75 \mathrm{a} / 40 \mathrm{a}\right)$ ergibt sich ein Risiko von $1 \times 10^{-6}$ bei ca. $0,0001 \mathrm{mg} / \mathrm{m}^{3}=0,1 \mu \mathrm{g} / \mathrm{m}^{3}$. Aus der aktuellen wissenschaftlichen Literatur lässt sich für Benzol eine aktuelle Innenraumluftbelastung, hier als 95. Perzentil in Wohninnenräumen von $4,5 \mu \mathrm{g} / \mathrm{m}^{3}$ abschätzen.

Vor diesem Hintergrund wird als vorläufiger Leitwert eine Konzentration von 4,5 $\mu \mathrm{g} \mathrm{Benzol} / \mathrm{m}^{3}$ festgelegt.

\section{Hinweise zur Messstrategie}

Der vorläufige Leitwert für Benzol wurde auf Basis der Deutsche Umweltstudie zur Gesundheit, GerES 2014-2017 [60] abgeleitet, so dass zur vergleichenden Beurteilung von Benzol ebenfalls die Probenahmestrategie zu verwenden ist, die bei der Erstellung des Datenkollektives zu Grunde gelegt wurde (Langzeitmessung über sieben Tage mittels Passivsammler unter üblichen Nutzungsbedingungen).

Für den Fall, dass von einer dauerhaft erhöhten Exposition gegenüber Benzol auszugehen ist, und diese nicht durch die Benzolkonzentration der Außenluft bedingt ist, sind ggf. expositionsmindernde Maßnahmen einzuleiten, deren Wirksamkeit zu überprüfen ist.

\section{Anmerkungen}

Der Textentwurf dieser Mitteilung wurde federführend von Hermann Fromme, Katrin Schröder, Malgorzata Debiak, Claudia Röhl, Thomas Lahrz, Martin Kraft und mit Beiträgen von Herrn Herbert Grams erstellt und vom Ausschuss für Innenraumrichtwerte im Oktober 2019 verabschiedet. Die Literaturrecherche wurde im Mai 2019 abgeschlossen.

Danksagung. Wir bedanken uns ganz herzlich für die wesentlichen Beiträge, die Herr Helmut Sagunski, der leider im Dezember 2018 verstorben ist, zur Entstehung dieses Beitrages geleistet hat. 


\section{Literatur}

1. 39. BImSchV (2010) Neununddreißigste Verordnung zur Durchführung des Bundes-Immissionsschutzgesetzes (Verordnung über Luftqualitätsstandards und Emissionshöchstmengen - 39 . BImSchV). Online: https://www.gesetze-im-internet.de/bimschv_39/.Zugegriffen: 17. Juni 2019

2. AGS (2012) Begründung zu Benzol in BekGS910. Stand: Mai 2012. Ausgabe: November 2012. Ausschuss für Gefahrstoffe. https://www.baua. de/DE/Angebote/Rechtstexte-und-TechnischeRegeln/Regelwerk/TRGS/pdf/910/910-benzol. pdf?_ blob=publicationFile\&v=2. Zugegriffen: 17 . Juni 2019

3. AIR (2015) Gesundheitliche Bewertung krebserzeugender Verunreinigungen der Innenraumluft - erste Ergänzung zum Basisschema. Ausschuss für Innenraumrichtwerte. Bundesgesundheitsbl 58:769-773.

4. ANSES (2012) Indoor Air Quality Guidelines (IAQGs). Agence National de Securité Sanitaire de I'Alimentation, I'Environnement et le Travail. https://www.anses.fr/en/content/indoor-air-qualityguidelines-iaqgs. Zugegriffen: 17. Juni 2019

5. Campagna M, Satta G, Campo L, Flore V, Ibba A, Meloni M, Tocco MG, Avataneo G, Flore C, Fustinoni S, Cocco P (2014) Analysis of potential influence factors on background urinary benzene concentration among a non-smoking, non-occupationally exposed general population sample. Int Arch Occup Environ Health 87: 793-799. Cancer Epidemiol Biomarkers Prev 15: 2246-2252.

6. Carlos-Wallace FM, Zhang L, Smith MT, Rader G, Steinmaus C (2016) Parental, in utero, and earlylife exposure to benzene and the risk of childhood leukemia: a meta-analysis. Am J Epidemiol 183:1-14

7. DECOS (2014) Benzene. Health-based recommended occupational exposure limit. Health Council of the Netherlands. https://www.healthcouncil. $\mathrm{nl} /$ documents/advisory-reports/2014/02/21/benzene-health-based-recommended-occupationalexposure-limit. Zugegriffen: 17. Juni 2019

8. DGUV (2018) Liste der krebserzeugenden, keimzellmutagenen und reproduktionstoxischen Stoffe (KMR-Liste). https://www.dguv.de/ifa/fachinfos/ kmr-liste/index.jsp. Zugegriffen: 17. Juni 2019

9. ECHA (2018) Opinion on scientific evaluation of occupational exposure limits for benzene. Adopted 9 March 2018. European Chemicals Agency. Committee for Risk Assessment. ECHA/RAC/0000000-1412-86-187/F. https://echa.europa.eu/ documents/10162/13641/benzene_opinion en.pdf/4fec9aac-9ed5-2aae-7b70-5226705358c7. Zugegriffen: 17. Juni 2019

10. EU-KOMM (2004) Richtlinie 2004/37/EG des Europäischen Parlaments und des Rates vom 29. April 2004 über den Schutz der Arbeitnehmer gegen Gefährdung durch Karzinogene oder Mutagene bei der Arbeit. Amtsblatt der Europäischen Union L 229/23. Online: https://eur-lex.europa.eu/LexUriServ/LexUriServ.do?uri=OJ:L:2004:229:0023:0034: DE:PDF. Zugegriffen: 17. Juni 2019

11. EU-SCOEL (Scientific Committee on Occupational Exposure Limits) (2016) Recommendation from the Scientific Committee on Occupational Exposure Limits for benzene.

12. Fiedler W, Janning M, Kebenko M, Weidmann J, Janjetovic S, Bokemeyer C (2014) Akute myeloische Leukämie - bestmögliche Behandlung. Hamb Arztebl 68:12-17
13. Filippini T, Hatch EE, Rothman KJ, Heck JE, Park AS, Crippa A, Orsini N, Vinceti M (2019) Association between Outdoor Air Pollution and Childhood Leukemia: A Systematic Review and DoseResponse Meta-Analysis. Environ Health Perspect 127:46002

14. Fromme $H$, Heitmann $D$, Dietrich $S$, Schierl $R$, Körner W, Kiranoglu M, Zapf A, Twardella D (2008) Raumluftqualität in Schulen - Belastung von Klassenräumen mit Kohlendioxid $\left(\mathrm{CO}_{2}\right)$, flüchtigen organischen Verbindungen (VOC), Aldehyden, Endotoxinen und Katzenallergenen. Gesundheitswesen 70:88-97

15. Grams H, Gierden E (2016) Folgeuntersuchung zum Einfluss von holzbefeuerten Kaminöfen auf die Qualität der. Innenraumluft Gefahrstoffe Reinhalt Luft 76:68-70

16. Greenlee WF, Gross EA, Irons RD (1981) Relationship between benzene toxicity and the disposition of 14C-labelled benzene metabolites in the rat. Chem Biol Interact 33:285-299

17. Hartwig A (2010) The role of DNA repair in benzene-induced carcinogenesis. Chem Biol Interact 184:269-272

18. Heck JE, He D, Contreras ZA, Ritz B, Olsen J, Hansen $J$ (2019) Parental occupational exposure to benzene and the risk of childhood and adolescent acute lymphoblastic leukaemia: a population-based study. Occup Environ Med 76:527-529

19. Heck JE, Park AS, Qiu J, Cockburn M, Ritz B (2014) Risk of leukemia in relation to exposure to ambient air toxics in pregnancy and early childhood. Int J Hyg Environ Health 217:662-668

20. Hofmann H, Erdmann G, Müller A (2014) Zielkonflikt energieeffiziente Bauweise und gute Raumluftqualität - Datenerhebung für flüchtige organische Verbindungen in der Innenraumluft von Wohn- und Bürogebäuden (Lösungswege). https://www.agoef.de/publikationen/publikationenagoef.html. Zugegriffen: 17. Juni 2019

21. Huang Z, Zhang Y, Yan Q, Zhang Z, Wang X (2016) Real-time monitoring of respiratory absorption factors of volatile organic compounds in ambient air by proton transfer reaction time-of-flight mass spectrometry. J Hazard Mater 320:547-555

22. IARC (2018) IARC Monographs Volume 120: Benzene. International Agency for Research on Cancer, Lyon, France. Online unter: http://publications.iarc. fr/576. Zugegriffen: 17. Juni 2019

23. Infante PF (2017) Residential Proximity to Gasoline Stations and Risk of Childhood Leukemia. Am J Epidemiol 185:1-4

24. IPCS (1993) BENZENE. Environmental Health Criteria 150. WHO, International Programme on Chemical Safety, Geneva, Switzerland. Online: http://www.inchem.org/documents/ehc/ehc/ ehc150.htm. Zugegriffen: 17. Juni 2019

25. Janitz AE, Campbell JE, Magzamen S, Pate A, Stoner JA, Peck JD (2017) Benzene and childhood acute leukemia in Oklahoma. Environ Res 158:167-173

26. Kim S, Vermeulen $R$, Waidyanatha $S$, Johnson $B A$, Lan Q, Smith MT, Zhang L, Li G, Shen M, Yin S, Rothman N, Rappaport SM (2006) Modeling human metabolism of benzene following occupational and environmental exposures.

27. Kirkeleit J, Riise T, Bjørge T, Christiani DC, Bråtveit M, Baccarelli A, Mattioli S, Hollund BE, Gjertsen BT (2018) Maternal exposure to gasoline and exhaust increases the risk of childhood leukemia in offspring - a prospective study in the Norwegian Mother and Child Study. Br J Cancer 119:10281035
28. Koh DH, Jeon HK, Lee SG, Ryu HW (2015) The relationship between low-level benzene exposure and blood cell counts in Korean workers. Occup Environ Med 72:421-427

29. Kraus T, Bader M, Klotz K, Weistenhöfer W, Drexler H, Hartwig A (2018) Addendum zu Benzol. The MAK Collection for Occu-pational Health and Safety 3(1):262-294. https:// doi.org/10.1002/3527600418.bb7143d0023

30. Lan Q, Zhang L, Li G, Vermeulen R, Weinberg RS, Dosemeci M, Rappaport SM, Shen M, Alter BP, Wu Y, Kopp W, Waidyanatha S, Rabkin C, Guo W, Chanock S, Hayes RB, Linet M, Kim S, Yin S, Rothman N, Smith MT (2004) Haematoxicity in workers exposed to low levels of benzene. Sci 306:1774-1776

31. LaSD (2009) Raumluftuntersuchungen in öffentlichen Gebäuden in Schleswig-Holstein. Teil 1: Hintergrundwerte für Schulen und Kindergärten (Schul- und Kindergartenstudie 2005 / 2007). Landesamt für soziale Dienste des Landes Schleswig-Holstein. Kiel. Online: https:// www.schleswig-holstein.de/DE/Fachinhalte/G/ gesundheitsschutz_umweltbezogen/Luft/ Downloads/studie_Raumluft_1_2009.pdf? blob=publicationFile\&v=6. Zugegriffen: 17 . Juni 2019

32. Li W, Schnatter AR (2018) Benzene risk assessment: does new evidence on myelodysplastic syndrome justify a new approach? Crit Rev Toxicol 48:417-432

33. Loomis D, Guyton KZ, Grosse Y, El Ghissassi F, Bouvard V, Benbrahim-Tallaa L, Guha N, Vilahur N, Mattock H, Straif K (2017) Carcinogenicity of benzene. Lancet Oncol 18:1574-1575

34. McKenzie LM, Allshouse WB, Byers TE, Bedrick EJ, Serdar B, Adgate JL (2017) Childhood hematologic cancer and residential proximity to oil and gas development. PLOS ONE 12:e170423

35. Medeiros Vinci R, Jacxsens L, Van Loco J, Matsiko E, Lachat C, de Schaetzen T, Canfyn M, Van Overmeire I, Kolsteren P, De Meulenaer B (2012) Assessment of human exposure to benzene through foods from the Belgian market. Chemosphere 88:1001-1007

36. Metzeler KH, Herold T, Rothenberg-Thurley M, Amler S, Sauerland MC, Görlich D, Schneider S, Konstandin NP, Dufour A, Bräundl K, Ksienzyk B, Zellmeier E, Hartmann L, Greif PA, Fiegl M, Subklewe M, Bohlander SK, Krug U, Faldum A, Berdel WE, Wörmann B, Büchner T, Hiddemann W, Braess J, Spiekermann K (2016) Spectrum and prognostic relevance of driver gene mutations in acute myeloic leukemia. Blood 128:686-698

37. Nagata $Y$ (2003) Measurement of odor threshold by triangle odor bag method. In: Odor measurement review. Office of Odor, Noise and Vibration. Environmental Management Bureau, Ministry of Environment, Tokyo, S 118-127. http://www.env. go.jp/en/air/odor/measure/02_3_2.pdf

38. Nomiyama K, Nomiyama H (1974) Respiratory retention, uptake and excretion of organic solvents in man. Int Arch Arbeitsmed 32:75-83

39. Pekari K, Vainiotalo S, Heikkilä P, Palotie A, Luotamo M, Riihimäki V (1992) Biological monitoring of occupational exposure to low levels of benzene. Scand JWork Environ Health 18:317-322

40. Qu Q, Melikian AA, Li G, Shore R, Chen L, Cohen $B$, Yin S, Kagan MR, Li H, Meng M, Jin X, Winnik W, LiY, Mu R, Li K (2000) Validation of biomarkers in humans exposed to benzene: Urine metabolites. Am J Ind Med 37:522-531

41. Qu Q, Shore R, Li G, Jin X, Chen L-C, Cohen B, Melikian AA, Eastmond D, Rappaport S, Li H, Rupa D, Waidyanatha S, Yin S, Yan H, Meng M, 
Winnik W, Kwok ESC, Li Y, Mu R, Xu B, Zhang X, Li K (2003) Validation and Evaluation of Biomarkers in Workers Exposed to Benzene in China. Health Effects Institute Research, Report 115, Boston, US, MA. https://www.healtheffects.org/system/files/ Qu.pdf

42. Qu Q, Shore R, Li G, Su L, Jin X, Melikian AA, Roy N, Chen LC, Wirgin I, Cohen B, Yin S, Li Y, Mu R (2005) Biomarkers of benzene: urinary metabolites in relation to individual genotype and personal exposure. Chem Biol Interact 153-154:85-95

43. Raaschou-Nielsen O, Hvidtfeldt UA, Roswall N, Hertel O, Poulsen AH, Sørensen M (2018) Ambient benzene at the residence and risk for subtypes of childhood leukemia, lymphoma and CNS tumor. Int J Cancer 143:1367-1373

44. Rappaport SM, Kim S, Lan Q, Li G, Vermeulen R, Waidyanatha S, Zhang L, Yin S, Smith MT, Rothman $\mathrm{N}$ (2010) Human benzene metabolism following occupational and environmental exposures. Chemico-Biol Interact 184:189-195

45. Rappaport SM, Kim S, Thomas R, Johnson BA, Bois FY, Kupper LL (2013) Low-dose metabolism of benzene in humans: science and obfuscation. Carcinogenesis 34:2-9

46. Rauma M, Boman A, Johanson G (2013) Predicting the absorption of chemical vapours. Adv Drug Deliver Rev 65:306-324

47. RIVM (2007) Health-based guidelines for the indoor environment. RIVM report 609021044/2007. APPENDIX A: Justification of the health-based guideline values for Chemicals. Online: https:// www.rivm.nl/bibliotheek/rapporten/609021044. pdf. Zugegriffen: 18. Oktober 2019

48. RIVM (2001) Re-evaluation of human-toxicological maximum permissible risk levels. RIVM Rapport 711701025. Appendix 3 Aromatic compounds. https://www.rivm.nl/bibliotheek/rapporten/711701025.pdf. Zugegriffen: 18. Okt. 2019

49. Rosenthal GJ, Snyder CA (1987) Inhaled benzene reduces aspects of cell-mediated tumor surveillance in mice. Toxicol Appl Pharmacol 88:35-43

50. Rothman N, Smith MT, Hayes RB, Li GL, Irons RD, Dosemeci M, Haas R, Stillman WS, Linet M, Xi LQ, Bechtold WE, Wiemels J, Campleman S, Zhang L, Quintana PJ, Titenko-Holland N, Wang YZ, Lu W, Kolachana P, Meyer KB, Yin S (1996) An epidemiologic study of early biologic effects of benzene in Chinese workers. Environ Health Perspect 104, Suppl 6: 1365-1370.

51. Rozen MG, Snyder CA, Albert RE (1984) Depression in B- and T-lymphocyte mitogen-induced blastogenesis in mice exposed to low concentrations of benzene. Toxicol Lett 20:343-349

52. Sabourin PJ, Sun JD, Birnbaum LS, Lucier G, Henderson RF (1989a) Effect of repeated benzene inhalation exposures on subsequent metabolism of benzene. Exp Pathol 37:155-157

53. Sabourin PJ, Bechtold WE, Griffith WC, Birnbaum LS, Lucier G, Henderson RF (1989b) Effect of exposure concentration, exposure rate, and route of administration on metabolism of benzene by $\mathrm{F} 344$ rats and B6C3F1 mice. Toxicol Appl Pharmacol 99:421-444

54. Setton E, Hystad P, Poplawski K, Cheasley R, Cervantes-Larios A, Keller CP, Demers PA (2013) Risk-based indicators of Canadians' exposures to environmental carcinogens. Environ Health 12:15

55. Spycher BD, Lupatsch JE, Huss A, Rischewski J, Schindera C, Spoerri A, Vermeulen R, Kuehni CE (2017) Parental occupational exposure to benzene and the risk of childhood cancer: A census-based cohort study. Environ Int 108:84-91
56. Tsai SP, Fox EE, Ransdell JD, Wendt JK, Waddell LC, Donnelly RP (2004) A hematology surveillance study of petrochemical workers exposed to benzene. Regul Toxicol Pharmacol 40:67-73

57. UBA (1991) Umwelt-Survey 1985/1986. Band Illc: Wohn-Innenraum: Raumluft. https://www. umweltbundesamt.de/publikationen/umweltsurvey-19851986-3. Zugegriffen: 17. Juni 2019

58. UBA (2008) Vergleichswerte für flüchtige organische Verbindungen (VOC und Aldehyde) in der Innenraumluft von Haushalten in Deutschland. Bundesgesundheitsbl 51:109-112

59. UBA (2018) Benzol im Jahr 2017. https://www. umweltbundesamt.de/themen/luft/luftschadstoffe/benzol. Zugegriffen: 17. Juni 2019

60. UBA (2019) Benzol in der Innenraumluft von Wohnungen. Persönliche Mitteilung

61. UfU (1994) Vorkommen von flüchtigen Luftverunreinigungen in Schulen und Kindergärten. Jahresbericht der Untersuchungsstelle für Umwelttoxikologie des Landes Schleswig-Holstein 1992/93, Kiel

62. US-ATSDR (2007) Toxicological Profile for Benzene. Agency for Toxic Substances and Disease Registry, Atlanta, GA, USA. Online: https://www.atsdr.cdc. gov/toxprofiles/tp.asp?id=40\&tid=14. Zugegriffen: 17. Juni 2019

63. US-EPA (2003) Toxicological review of Benzene. Integrated Risk Information System (IRIS), IRIS. U.S Environmental Protection Agency. Online: https:// cfpub.epa.gov/ncea/iris2/chemicalLanding. cfm?\&substance_nmbr=276. Zugegriffen: 17 . Juni 2019

64. Vinceti M, Rothman KJ, Crespi CM, Sterni A, Cherubini A, Guerra L, Maffeis G, Ferretti E, Fabbi S, Teggi $S$, Consonni D, De Girolamo G, Meggiato A, Palazzi $G$, Paolucci P, Malagoli C (2012) Leukemia risk in children exposed to benzene and PM10 from vehicular traffic: a case-control study in an Italian population. Eur J Epidemiol 27:781-790

65. Weisel CP (2010) Benzene exposure: an overview of monitoring methods and their findings. Chem Biol Interact 184:58-66

66. WHO (2010) WHO guidelines for indoor air quality: selected pollutants. World Health Organization. Regional Office for Europe, Copenhagen. https:// apps.who.int/iris/handle/10665/260127. Zugegriffen: 17. Juni 2019

67. Zhu J, Wong SL, Cakmak S (2013) Nationally representative levels of selected volatile organic compounds in Canadian residential indoor air: population-based survey. Environ Sci Technol 47:13276-13283 